\title{
Correlation between inflammatory parameters and pituitary-thyroid axis in patients with COVID-19
}

\author{
Verónica llera $\mathbb{1}^{1} \cdot$ Laura C. Delfino $^{1}$ - Anabela Zunino ${ }^{1}$ Patricia Glikman ${ }^{1}$ - Mónica Drnovsek ${ }^{1}$ Adriana Reyes ${ }^{1}$. \\ Alicia Dios $^{1} \cdot$ Javier Toibaro ${ }^{2} \cdot$ Valeria Pachioli $^{2} \cdot$ Nayla Lannes $^{1} \cdot$ Alberto Guida $^{2} \cdot$ Alicia Gauna $^{1}$
}

Received: 25 May 2021 / Accepted: 31 August 2021 / Published online: 13 September 2021

(C) The Author(s), under exclusive licence to Springer Science+Business Media, LLC, part of Springer Nature 2021

\begin{abstract}
Purpose Inflammation plays a critical role in the progression of COVID-19. Nonthyroidal illness syndrome (NTIS) has been increasingly recognized in affected patients. We aim to evaluate the correlation of thyroid hormones with markers of inflammation and association with disease outcome in hospitalized patients with COVID-19, and in two profiles of NTIS (low T3-normal/low FT4 vs. low T3-high FT4).

Methods consecutive patients admitted to a nonintensive care unit for COVID-19 were recruited. Infection was mild in $22 \%$, moderate in $27.1 \%$ and severe in $50.8 \% ; 7.41 \%$ died. T4, T3, FT4, FT3, and their ratios (T3/T4, FT3/FT4) were correlated with albumin, ferritin, fibrinogen, erythrocyte sedimentation rate (ESR), C-reactive protein (CRP), lactate dehydrogenase (LDH), and D-dimer.

Results Fifty five patients (50.9\% men, median age 56 years) were included. Albumin correlated positively with T3 and hormones ratios, but negatively with FT4. T3, FT3, T3/T4, and FT3/FT4 correlated inversely with ferritin, fibrinogen, ESR, CRP, LDH, and D-dimer. FT4 showed direct correlation with fibrinogen and ESR. T3/T4 was lower in severe compared to mild/moderate disease [7.5 (4.5-15.5) vs. 9.2 (5.8-18.1); $p=0.04$ ], and lower in patients who died than in those discharged [5 (4.53-5.6) vs. 8.1 (4.7-18.1); $p=0.03$ ]. A low T3/high FT4 profile was associated with lower albumin, higher ferritin, and severity.

Conclusion In this cohort, thyroid hormones correlated with inflammation and outcome. T3 and T3/T4 correlated inversely with inflammatory markers; a low T3/T4 ratio was associated with severity and poor prognosis. Patients with low T3 but high FT4 had higher ferritin, lower albumin, and more severe disease at presentation.
\end{abstract}

Keywords COVID-19 - Inflammation · Thyroid hormones · Nonthyroidal illness syndrome

\section{Introduction}

The outbreak of coronavirus disease 2019 (COVID-19), caused by severe acute respiratory syndrome coronavirus 2 (SARS-CoV2), has rapidly spread worldwide and led to the declaration of Public Health Emergency of International Concern by the World Health Organization [1, 2]. SARS$\mathrm{CoV}-2$ has a wide spectrum of clinical severity, ranging

Verónica Ilera

veronicailera@gmail.com

1 Endocrinology Division, Hospital José María Ramos Mejía, Ciudad Autónoma de Buenos Aires, Argentina

2 Internal Medicine Division, Hospital José María Ramos Mejía, Ciudad Autónoma de Buenos Aires, Argentina from a- or pauci-symptomatic presentation to diseasespecific mortality [3]. COVID-19 has the potential to cause upper respiratory tract, pulmonary and systemic inflammation, determining multi-organ dysfunction especially in frail patients [4].

Both direct (i.e., caused by the virus infection of the target cells) and indirect injury (i.e., through abnormal immune inflammatory responses to the virus and likely involving the coagulation, cytokine and complement systems) have been linked to the wide clinical expression spectrum and multisystem organ failure of COVID-19 and SARS [5-9]. Angiotensin-converting enzyme 2 (ACE2), the functional receptor for SARS-CoV-2, plays a role in the pathogenesis of COVID-19. ACE2 expression is present in many endocrine organs, including the thyroid gland [10]. Increasing evidence suggests that COVID-19 affects thyroid function [11]. 
The nonthyroidal illness syndrome (NTIS) comprises a constellation of alterations in the central component of the hypothalamus-pituitary-thyroid (HPT) axis and changes in thyroid hormones (TH) metabolism in a variety of TH target organs [12]. NTIS can occur in several acute or chronic systemic diseases including cardiovascular, respiratory, infectious diseases, and cancer [13]. The most typical hormonal changes are low plasma T3, low or normal plasma $\mathrm{T} 4$, or elevated plasma reverse (rT3), in the presence of normal or slightly decreased TSH [13]. Cytokines, released during illness, are considered a major determinant of NTIS since they affect a variety of genes involved in TH metabolism [12]. Chen reported that NTIS could underlie the hormonal changes of at least $30 \%(15 / 50)$ of hospitalized patients with COVID-19 [14]. A study by Khoo et al. [15] detected that patients with COVID-19 had lower admission TSH and FT4 levels compared to those without COVID-19. Ten patients with COVID-19 in a study by Lui et al. [16] were found to have isolated low FT3, with normal TSH and FT4 levels, suggesting a possible NTIS; FT3 in the study showed a decreasing trend with worsening clinical severity of the disease. Furthermore, an independent inverse correlation between erythrocyte sedimentation rate (ESR) and FT3/FT4 ratio was also demonstrated in the study by Lui et al. [16] which suggested the potential effect of systemic inflammation on deiodinase activity [17]. Our group studied a population of hospitalized patients with COVID-19 and found low T3 in 42/ $57(73,7 \%)$. Most of them represented classic NTIS profile, with low/normal FT4 and normal TSH, but $11 / 42$ (26\%) of the patients with low T3 had high FT4 (data submitted for publication). Whether this represents a new NTIS profile is not yet fully clarified.

In the present study, we aimed to evaluate the correlation of $\mathrm{TH}$ with biochemical markers of inflammation and association with disease outcome in hospitalized patients with COVID-19. We also evaluated these parameters in the two profiles of NTIS (low T3-normal/low FT4 vs. low T3-high FT4).

\section{Material and methods}

This was an observational, cross sectional, single-center cohort study performed between August and November 2020. Consecutive patients admitted to the medical clinic ward of Jose María Ramos Mejía Hospital (a nonintensive care unit) were recruited. The presence of SARS-CoV2 was confirmed in all patients by RT-PCR from the nasopharyngeal swab. Patients were included if they were 18 years of age or older, were willing to participate in the study and signed informed consent. Patients were excluded if: (1) blood samples could not be obtained within $48 \mathrm{~h}$ of admission, (2) they had received previous medication that could potentially impact on thyroid function such as systemic steroids or heparin, and (3) written informed consent could not be obtained. Clinical and demographic data were extracted from patient records. Data regarding major comorbidities, vital signs, baseline oxygen saturation by pulse oximetry, chest X-ray and chest CT scan, when performed, were registered.

Each patient had blood tests upon admission before the initiation of treatments of COVID-19. Complete blood count and biochemical parameters such as urea, creatinine, fasting glucose, total cholesterol, aspartate and alanine aminotransferase, albumin (reference range [RR] 3.5-5.2 g/ $\mathrm{dl}$ ), ferritin (RR 20-159 ng/ml), fibrinogen (RR 150-450 mg\%), ESR (RR 1-15 mm/h), C-reactive protein (CRP) (RR 0-5.0 mg/l), lactate dehydrogenase (LDH) (RR 230-480 UI/l), and D-dimer (RR 0-500 ng/ml) were analyzed after a $12 \mathrm{~h}$ fasting. $\mathrm{TH}$ [thyrotropin (TSH: 0.5-4.0 mIU/ml), total T4 (T4: 5-11.5 $\mu \mathrm{g} / \mathrm{dl})$, free T4 (FT4: 0.8-1.4 ng/dl), total T3 (T3: 85-175 ng/dl), free T3 (FT3: $1.9-4.1 \mathrm{pg} / \mathrm{ml}$ )], and thyroid peroxidase antibody (TPOAb: $<20 \mathrm{IU} / \mathrm{ml}$ ) were measured with chemiluminescence assay IMMULITE 2000. Samples were obtained within $48 \mathrm{~h}$ of admission.

COVID-19 severity was classified according to the "Chinese Clinical Guidance for COVID-19 Pneumonia Diagnosis and Treatment (7th edition)" published by the Chinese National Health Commission [18]. Mild disease was defined by mild clinical symptoms without manifestations of pneumonia on imaging. Moderate disease was defined by fever and respiratory symptoms, and manifestations of pneumonia on imaging. Severe disease was defined by any of the following: respiratory rate $\geq 30 / \mathrm{min}$, $\mathrm{SpO} 2 \leq 93 \%$ at rest, and $>50 \%$ progression in $48 \mathrm{~h}$ on imaging. A favorable evolution was considered when patients did not have high oxygen requirements or were not admitted to the intensive care unit.

The study followed the principles in the Declaration of Helsinki and was approved by the Institutional Review Board of the Jose María Ramos Mejía Hospital. All patients provided written informed consent.

\section{Statistical analysis}

All data were analyzed using Statistical Package for Social Sciences (SPSS) version 21.0 software (SPSS Inc., Chicago, IL, United States); any $p$ value $<0.05$ was regarded as statistically significant. Categorical data were described using frequencies and percentages. Continuous data were described using median and range. The distribution of baseline characteristics across categories of the exposure variable was evaluated using nonparametric statistics. The proportions were compared with chi-square test or Fisher's exact test. Continuous variables were tested by Wilcoxon rank-sum test. Spearman correlations were used to assess the relationship between continuous variables. 
Table 1 Correlation between thyroid hormones and inflammatory parameters

\begin{tabular}{|l|l|l|l|l|l|l|}
\hline & \multicolumn{6}{|c|}{ Correlations (p<0.05) } \\
\hline & T4 & FT4 & T3 & FT3 & T3/T4 & FT3/FT4 \\
\hline Albumin & & R -0.55 & R 0.61 & & R 0.64 & R 0.56 \\
\hline Ferritin & & & & R -0.49 & R -0.5 & R -0.47 \\
\hline Fibrinogen & & R 0.41 & R -0.56 & R -0.42 & R -0.69 & R -0.54 \\
\hline ESR & R 0.33 & R 0.47 & & R -0.35 & R -0.49 & R -0.46 \\
\hline CRP & & & & & R -0.35 & \\
\hline LDH & & & & R -0.37 & & R -0.43 \\
\hline D- Dimer & & & R - 0.47 & R -0.42 & R -0.49 & R - 0.4 \\
\hline
\end{tabular}

In light blue, direct correlations. In orange, inverse correlations

\section{Results}

The final study population included 55 patients: 28 (50.9\%) men and $29(49.1 \%)$ women, with a median age of 56 years (range 21-89y). Patients were classified according to the severity of the infection as mild in $22 \%$ of the cases, moderate in $27.1 \%$ and severe in $50.8 \%$. $84.7 \%$ were considered to have a favorable evolution, while $3.4 \%$ had high oxygen requirements and $11.9 \%$ were admitted to the intensive care unit for mechanical ventilation. Four patients (7.41\%) of the total population died.

We found correlation between $\mathrm{TH}$ and $\mathrm{TH}$ ratios (T3/T4, FT3/FT4) with several inflammatory parameters (Table 1). Serum albumin correlated positively with $\mathrm{T} 3$ and $\mathrm{TH}$ rations, but negatively with FT4. We observed an inverse correlation between ferritin, fibrinogen, ESR, CRP, LDH, and D-dimer with T3, FT3. The magnitude of this correlation was even more important with T3/T4 and FT3/FT4 ratios. On the other hand, T4, and specially FT4, showed a direct correlation with fibrinogen and ESR.

When we analyzed TH levels according to severity of the disease and to clinical evolution of the patients, we observed that T3/T4 ratio was significantly lower in those patients with a severe form of the disease compared with those with mild/moderate infection [7.5 (4.5-15.5) vs. 9.2 $(5.8-18.1) ; p=0.04]$ (Fig. 1a). Also, T3/T4 ratio was significantly lower in patients who died than in patients who were finally discharged [5 (4.53-5.6) vs. 8.1 (4.7-18.1); $p=0.03$ ] (Fig. 1b).

Forty patients $(72.7 \%)$ had low T3 levels. Thirty of them had normal or low levels of FT4, with normal TSH, corresponding to a classic NTIS profile, whereas 10 had high
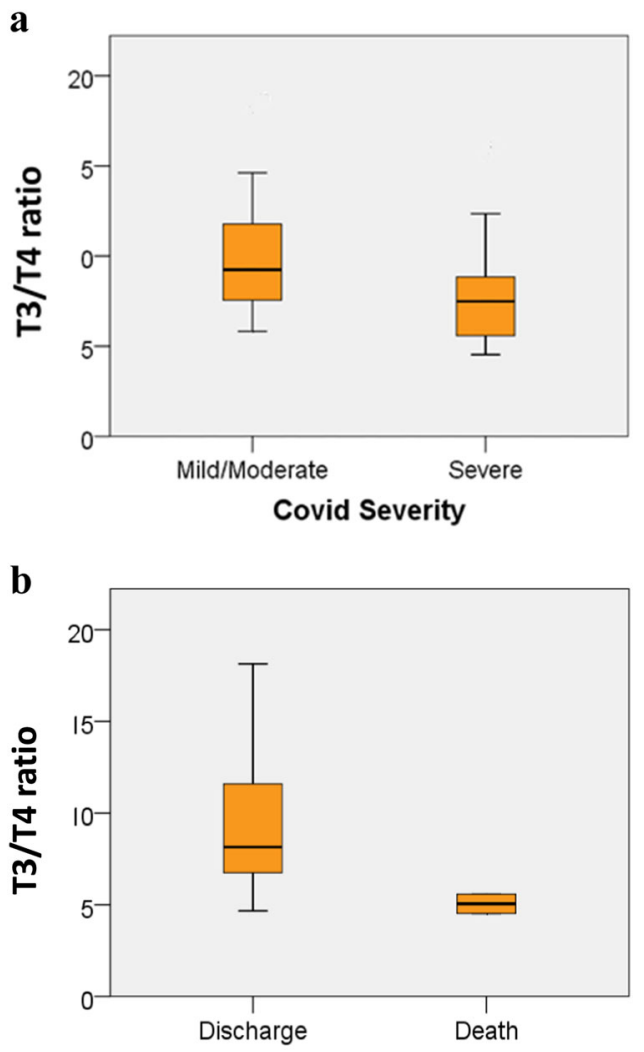

Final Outcome

Fig. 1 Thyroid hormones ratios according to severity of the disease and final outcome. a T3/T4 ratio in mild/moderate vs. severe disease. b T3/T4 ratio in patients finally discharged vs. those who died

FT4 levels with normal TSH. We observed that those with high FT4 had lower albumin $[3.4 \mathrm{~g} / \mathrm{dl}(3-4)$ vs. $3.7 \mathrm{~g} / \mathrm{dl}$ $(3-4) ; p=0.03]$, higher ferritin [1202 ng/ml (930-7127) vs. 
$\mathbf{a}$

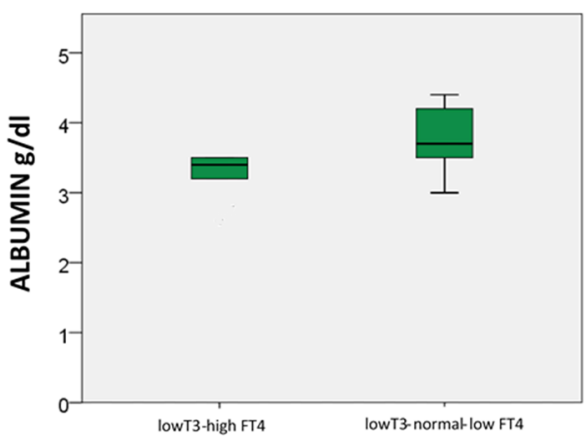

b

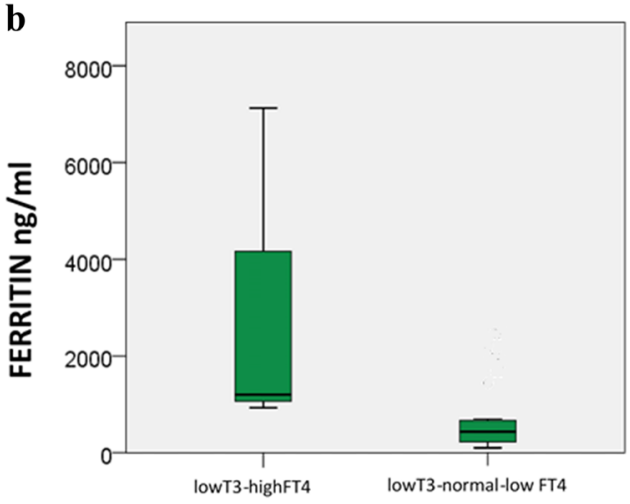

Fig. 2 Albumin and ferritin levels in patients with low T3 according to FT4. a Albumin levels in those with high FT4 vs. those with normal/ low FT4. b Ferritin levels in those with high FT4 vs. those with normal/low FT4

$435 \mathrm{ng} / \mathrm{ml}$ (101-2232); $p<0.05$ ] (Fig. 2a, b) and tendency to higher fibrinogen [681 $\mathrm{mg} \%(583-798)$ vs. $508 \mathrm{mg} \%$ $(307-807) ; p=0.06]$ than those with normal/low FT4. Also, no patient with mild disease at presentation had high FT4.

\section{Discussion}

In this study, we observed that, among patients with COVID-19 infection, TH and their ratios correlated with different parameters of inflammation. T3, FT3, T3/T4, and FT3/FT4 showed an inverse correlation with ferritin, fibrinogen, ESR, CRP, LDH, and D-dimer, whereas FT4 was directly correlated with fibrinogen and ESR.

Accumulating evidence has suggested that inflammatory responses play a critical role in the progression of COVID19. Inflammatory responses triggered by rapid viral replication of SARS-CoV-2 and cellular destruction can recruit macrophages and monocytes and induce the release of cytokines and chemokines. These cytokines and chemokines then attract immune cells and activate immune responses, leading to cytokine storms and aggravations. Several inflammatory markers such as procalcitonin, CRP, ESR, IL-6 have been reported to be significantly associated with the severity of COVID-19 [19]. NTIS presents as abnormal thyroid function in serious diseases other than thyroid disorders, including infection [13]. It is well established that NTIS is a consequence of an acute phase response to severe systemic illness or macronutrient restriction and usually presents as decreased plasma T3 level, or low or normal T4 and TSH levels. Cytokines, released during illness, are considered a major determinant of NTIS since they affect a variety of genes involved in TH metabolism [12].

Some studies have analyzed the thyroid function in COVID-19 patients and found patterns suggestive of NTIS [14-16, 20], but very few have reported an association with markers of inflammation. Lui et al. found that higher CRP level was independently associated with low FT3. FT3 and FT3/FT4 ratio showed an inverse correlation with CRP, similar to our findings [16]. Wang et al. identified increased levels of leukocytes, neutrophils, CRP and procalcitonin, and decreased level of lymphocytes in thyroid dysfunction group [20]. They defined thyroid dysfunction as any abnormality in TT3, TT4, or TSH. These findings, along with ours, suggest the potential effect of systemic inflammation on deiodinase activity. Systemic inflammation, (represented by higher inflammatory markers), which in turn is associated with systemic tissue injury, lead to reduce deiodinase activity. This results in decreased conversion of T4 to T3, leading to low FT3 $[12,21]$.

Data have shown that IL-6 levels are also significantly higher in COVID-19-patients with a severe status compared with those with a non-severe condition, so IL-6 is a prognostic marker in serious COVID-19 cases [22]. Moreover, serum IL-6 is also often elevated in NTIS (the exact mechanism is not defined yet), and its level is inversely related to T3 levels in NTIS patients. Lania et al. [23] also found that thyroid dysfunction, particularly thyrotoxicosis, was associated with a high level of IL-6 in patients with SARS-CoV-2 infection. Unfortunately, we were not able to determine IL-6 levels in our cohort.

Reduced T3 concentration has been related to mortality in patients with chronic renal failure, acute myocardial infarction, liver failure and surgical sepsis. In COVID-19 patients, low FT3 may also have prognostic significance. A previous retrospective study showed that COVID-19 patients who died had lower FT3 on admission compared with the survivors [4]. Chen et al. reported that the degree of the decreases in TT3 levels was positively correlated with the severity of the disease [14], while Gao et al. informed that reduced FT3 independently predicted all-cause mortality of patients with severe COVID-19 [24]. In a similar way, we observed that $\mathrm{T} 3 / \mathrm{T} 4$ ratio was significantly lower in those patients with a severe form of the disease and in patients who eventually died. Interestingly, we also noticed that a group of patients with low FT3 had high FT4. None 
of them presented with a mild form of the disease and FT4 showed positive correlation with some inflammatory parameters. Mercke et al. demonstrated that low FT3 levels and high FT4 were associated with higher all-cause and cardiovascular mortality in Caucasians undergoing angiography, independent of age and sex [25]. A possible explanation could be more profound alterations in deiodinase activity associated with inflammation.

Some strengths of this study include that all patients were recruited prospectively, and that they were evaluated before starting any specific medication that could affect thyroid hormone axis. Heparin has been largely used in COVID-19 and blood samples from heparin-treated patients have increased lipoprotein lipase activity generating nonesterified fatty acids (NEFA). NEFA can interfere in free thyroid hormone assays displacing $\mathrm{T} 4$ from binding proteins and causing spuriously high values. Corticosteroids, which are employed to overcome the inflammatory response caused by COVID-19, can affect TH by several pathways: reducing TSH, reducing levels of thyroxinebinding globulin and increasing free $\mathrm{T} 4$, and inhibiting thyroid hormone conversion from T4 to T3 [26]. Limitations could be related that it was a single-center protocol, and the lack of a control group without COVID-19 infection. We do not know if our results could be expected to be similar in hospitalized patients for other critical diseases. Unfortunately, we could not have a control population because at the moment the study was undertaken, admissions at internal medicine ward or intensive care unit of our institution were only related to COVID-19 patients.

In conclusion, in this cohort of hospitalized patients with COVID-19 infection, both TH (T3, T4, and free fractions) correlated, in an opposite way, with inflammation parameters and worse clinical outcome. In particular, T3 and T3/T4 ratio correlated inversely with markers of inflammation, whereas a low T3/T4 ratio was associated with severity and poor prognosis. Of note, those with low FT3 and high FT4 profile showed lower albumin, higher ferritin, and more severe disease at presentation. Further longitudinal studies are needed to determine the full impact of COVID-19 on the HPT axis.

\section{Data availability}

Upon request the authors are prepared to send relevant documentation or data in order to verify the validity of the results presented.

Author contributions All authors contributed to the study conception and design. Material preparation, data collection, and analysis were performed by V.I., A.Z. and L.D. The first draft of the paper was written by V.I. and all authors commented on previous versions of the paper. All authors read and approved the final paper.

\section{Compliance with ethical standards}

Conflict of interest The authors declare no competing interests.

Ethics approval All procedures performed in this study involving human participants were in accordance with the ethical standards of the institutional and/or national research committee and with the 1964 Helsinki Declaration and its later amendments or comparable ethical standards. The study was approved by the Bioethics Committee of the José María Ramos Mejía Hospital.

Informed consent All patients provided written informed consent.

Publisher's note Springer Nature remains neutral with regard to jurisdictional claims in published maps and institutional affiliations.

\section{References}

1. F. Wu, S. Zhao, B. Yu et al. A new coronavirus associated with human respiratory disease in China. Nature 579, 265-269 (2020). https://doi.org/10.1038/s41586-020-2008-3

2. R. Horton, Offline: 2019-nCoV outbreak-early lessons. Lancet 395(10221), 322 (2020). https://doi.org/10.1016/S0140-6736(20) 30212-9

3. C. Huang, Y. Wang, X. Li et al. Clinical features of patients infected with 2019 novel coronavirus in Wuhan, China. Lancet 395:497-506 (2020). https://doi.org/10.1016/S0140-6736(20) 30183-5

4. T. Chen, D. Wu, H. Chen et al. Clinical characteristics of 113 deceased patients with coronavirus disease 2019: retrospective study. BMJ 368, m1091 (2020). https://doi.org/10.1136/bmj. m1091

5. M. Ackermann, S.E. Verleden, M. Kuehnel et al. Pulmonary vascular endothelialitis, thrombosis, and angiogenesis in Covid19. N. Engl J. Med. 383(2):120-128 (2020). https://doi.org/10. 1056/NEJMoa2015432

6. E.M. Dufort, E.H. Koumans, E.J. Chow et al. Multisystem inflammatory syndrome in children in New York State. N. Engl J. Med. 383(4), 347-358 (2020). https://doi.org/10.1056/NEJMoa 2021756

7. F. Coperchini, L. Chiovato, L. Croce, F. Magri, M. Rotondi, The cytokine storm in COVID-19: An overview of the involvement of the chemokine/chemokine-receptor system. Cytokine Growth Factor Rev. 53, 25-32 (2020). https://doi.org/10.1016/j.cytogfr. 2020.05 .003

8. A. Java, A.J. Apicelli, M.K. Liszewski et al. The complement system in COVID-19: friend and foe? JCI Insight 5(15), 140711 (2020). https://doi.org/10.1172/jci.insight.140711

9. Y. Guo, C. Korteweg, M.A. McNutt, J. Gu, Pathogenetic mechanisms of severe acute respiratory syndrome. Virus Res. 133 (1), 4-12 (2008). https://doi.org/10.1016/j.virusres.2007.01.022

10. R. Pal, M. Banerjee, COVID-19 and the endocrine system: exploring the unexplored. J. Endocrinol. Investig. 43, 1027-1031 (2020). https://doi.org/10.1007/s40618-020-01276-8.

11. L. Scappaticcio, F. Pitoia, K. Esposito, A. Piccardo, P. Trimboli, Impact of COVID-19 on the thyroid gland: an update. Rev. Endocr. Metab. Disord. Nov. 25, 1-13 (2020). https://doi.org/10. 1007/s11154-020-09615-Z

12. E.M. de Vries, E. Fliers, A. Boelen, The molecular basis of the nonthyroidal illness syndrome. J. Endocrinol. 225(3), R67-R81 (2015). https://doi.org/10.1530/JOE-15-0133 
13. E. Fliers, A.C. Bianco, L. Langouche, A. Boelen, Thyroid function in critically ill patients. Lancet Diabetes Endocrinol. 3(10), 816-825 (2015). https://doi.org/10.1016/S2213-8587(15)00225-9

14. M. Chen, W. Zhou, W. Xu, Thyroid function analysis in 50 patients with COVID-19: a retrospective study. Thyroid 31(1), 8-11 (2021). https://doi.org/10.1089/thy.2020.0363

15. B. Khoo, T. Tan, S.A. Clarke et al. Thyroid function before, during and after COVID-19. J. Clin. Endocrinol. Metab. 106(2), e803-e811 (2021). https://doi.org/10.1210/clinem/dgaa830

16. D.T.W. Lui, C.H. Lee, W.S. Chow et al. Thyroid dysfunction in relation to immune profile, disease status and outcome in 191 patients with COVID-19. J. Clin. Endocrinol. Metab. 106(2), e926-e935 (2021). https://doi.org/10.1210/clinem/dgaa813

17. W. Chen, Y. Tian, Z. Li, J. Zhu, T. Wei, J. Lei, Potential interaction between SARS-CoV-2 and thyroid: a review. Endocrinology 162 (3), bqab004 (2021). https://doi.org/10.1210/endocr/bqab004

18. National Health Commission of the people's Republic of China. Chinese Clinical Guidance for COVID-19 Pneumonia Diagnosis and Treatment, 7th edn. Accessed 23 Aug 2020. http://kjfy. meetingchina.org/msite/news/show $/ \mathrm{cn} / 3337 . \mathrm{html}$

19. F. Zeng, Y. Huang, Y. Guo et al. Association of inflammatory markers with the severity of COVID-19: a meta-analysis. Int J. Infect. Dis. 96, 467-474 (2020). https://doi.org/10.1016/j.ijid. 2020.05.055

20. W. Wang, X. Su, Y. Ding, W. Fan, W. Zhou, J. Su, Z. Chen, H. Zhao, K. Xu, Q. Ni, X. Xu, Y. Qiu, L. Teng, Thyroid function abnormalities in COVID-19 patients. Front. Endocrinol. 11, 623792 (2021). https://doi.org/10.3389/fendo.2020.623792

21. A. Mancini, C. Di Segni, S. Raimondo, G. Olivieri, A. Silvestrini, E. Meucci, D. Currò, Thyroid hormones, oxidative stress, and inflammation. Mediators Inflamm. 2016, 6757154 (2016). https:// doi.org/10.1155/2016/6757154

22. G. Speer, P. Somogyi, Thyroid complications of SARS and coronavirus disease 2019 (COVID-19). Endocr. J. 68(2), 129-136 (2021). https://doi.org/10.1507/endocrj.EJ20-0443

23. A. Lania, M.T. Sandri, M. Cellini, M. Mirani, E. Lavezzi, G. Mazziotti, Thyrotoxicosis in patients with COVID-19: the THYRCOV study. Eur. J. Endocrinol. 183(4), 381-387 (2020). https://doi.org/10.1530/EJE-20-0335

24. W. Gao, W. Guo, Y. Guo et al. Thyroid hormone concentrations in severely or critically ill patients with COVID-19. J. Endocrinol. Investig. 44, 1031-1040 (2021). https://doi.org/10.1007/s40618020-01460-w

25. A. Merke, J. Merke, G. Silbernagel, W. März, Free thyroid hormones and mortality in caucasians undergoing angiography: the ludwigshafen risk and cardiovascular healtH (LURIC) study. Endocr. Pract. 23(3), 288-298 (2017). https://doi.org/10.4158/ EP161217.OR

26. P. Trimboli, C. Camponovo, L. Scappaticcio, G. Bellastella, A. Piccardo, M. Rotondi, Thyroid sequelae of COVID-19: a systematic review of reviews. Rev. Endocr. Metab. Disord. 22(2), 485-491 (2021). https://doi.org/10.1007/s11154-021-09653-1 